\title{
Approach for Identification of Geometry Change on Cultural Heritage Surface
}

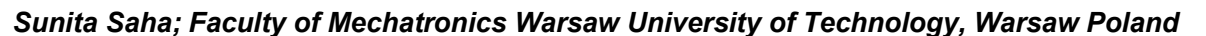

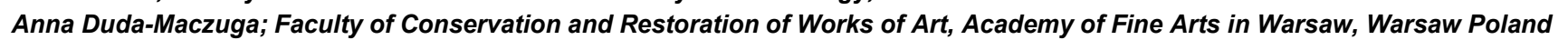

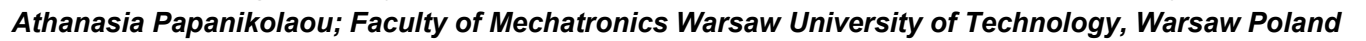

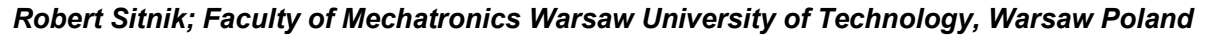

\begin{abstract}
Application of optical metrology techniques in the collection of surface data and its $3 D$ representation can improve the digital documentation of the conservation and restoration process of artworks. The tracking of induced change after the restoration process on cultural heritage (CH) surfaces involves a computational analysis of surface geometry. In the analysis, the conservation scientists were interested to see what impact the fillings of some holes have on its nearby surrounding during the reconstruction. In theory, the loss compensation method for stone should allow conservators to make a filling that only exists in the place of the void, but it is highly unlikely to make a filling that will only adhere to the substrate at the void site and not protrude elsewhere. According to the conservator scientists, we proposed an approach of local geometry changes to identify and visualize changes and presented the outcome through a local neighborhood distance histogram. This analysis will give us overall surface change considering each surface point and its respective neighborhood points and what impact it faced due to the reconstruction process. The work is also focused on developing the representation of each type of loss compensation method to make it more objective according to a restorer's point of view and simplify their work visibility.
\end{abstract}

Keywords: Computational Analysis, Cultural Heritage, 3D Scanning, Restoration, Surface Geometry Change, Visualization.

\section{Introduction}

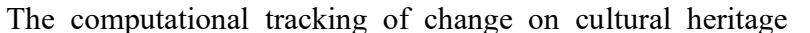

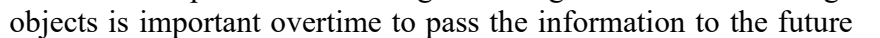

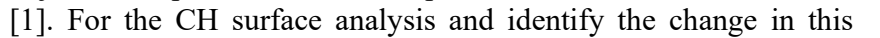
work, we considered a sculpture of a 'Lying Lion' shown in Figure $\square$

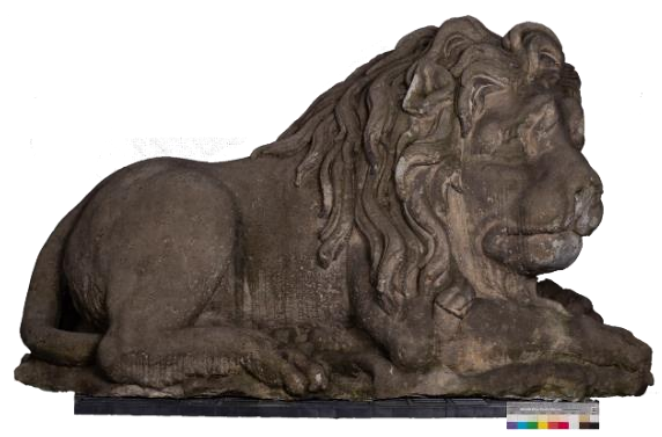

Figure 1. The chosen object, right side view before conservation (Photograph by Agnieszka Leszkowicz, Barbara Wabnik, Przemysław Wysk, under the supervision of Roman Stasiuk)
The sculpture of the 'Lying Lion' is one of two statues

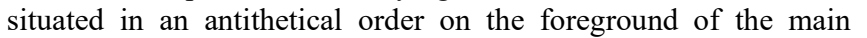
entrance of the Radziwiłł Palace in Nieborów. It was sculpted

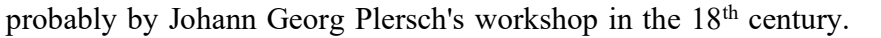

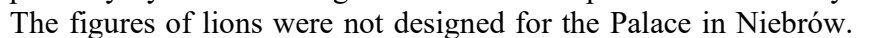

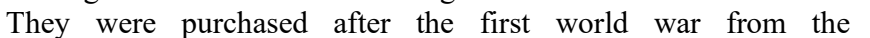

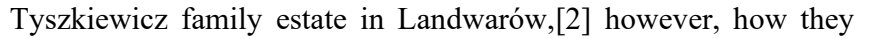

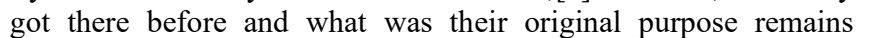
पामापा

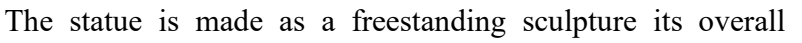

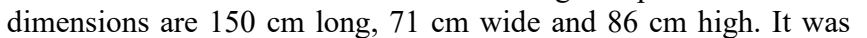

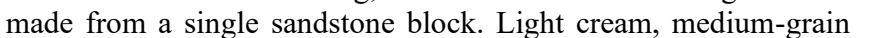

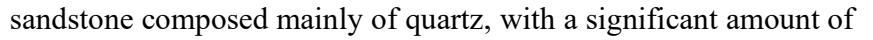

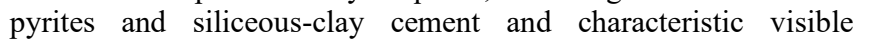

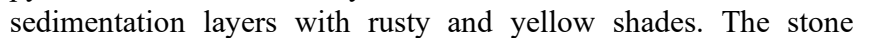

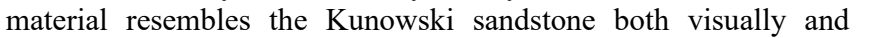
पापाणाणा

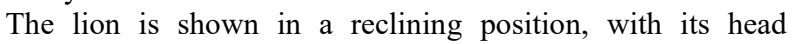

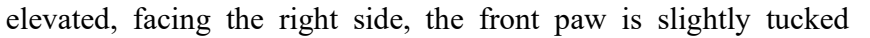

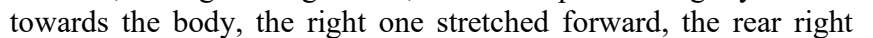

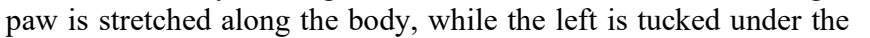

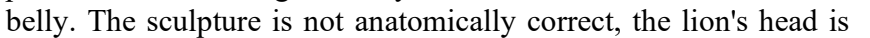

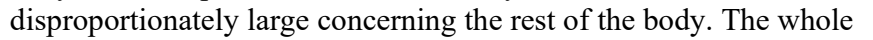

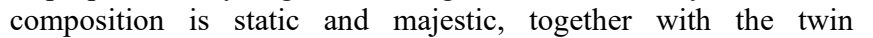

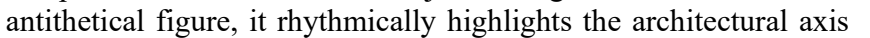

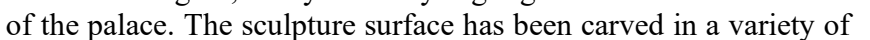

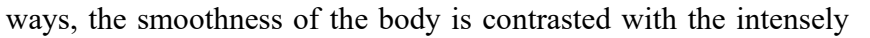

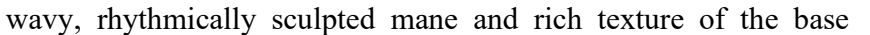

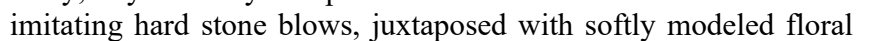
पण口पाणाण

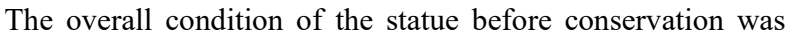

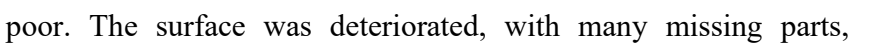

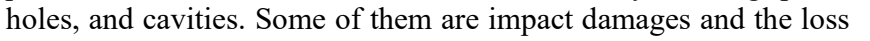

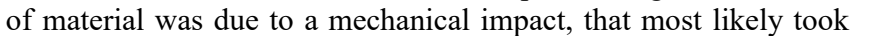

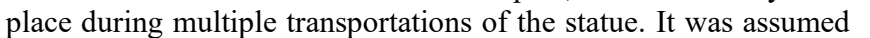

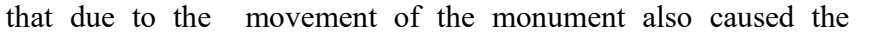

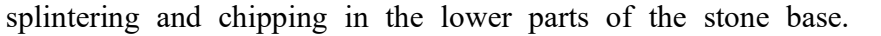

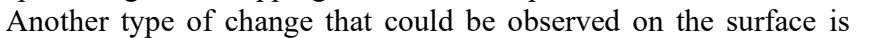

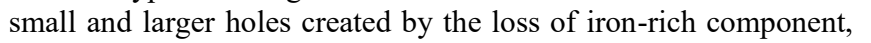

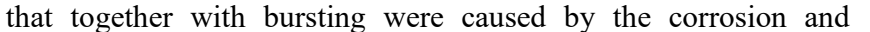

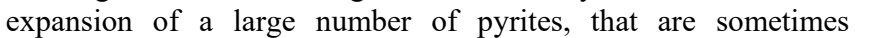

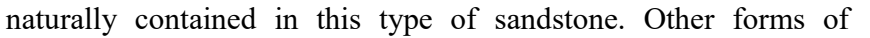

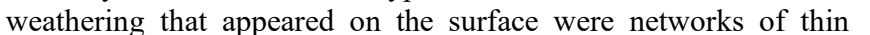

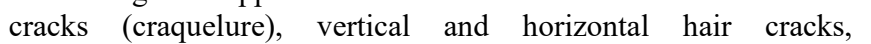

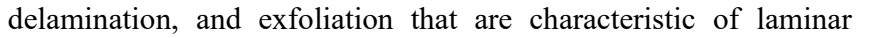

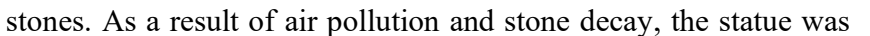

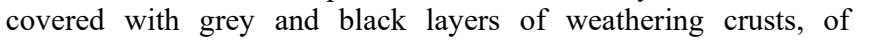

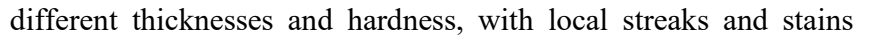
ए

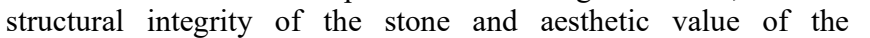




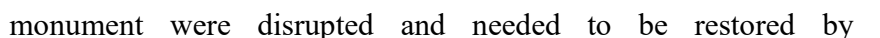

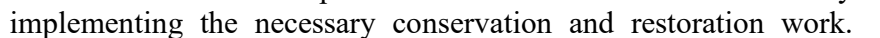

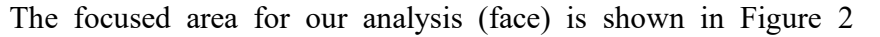

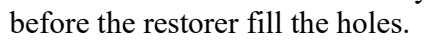

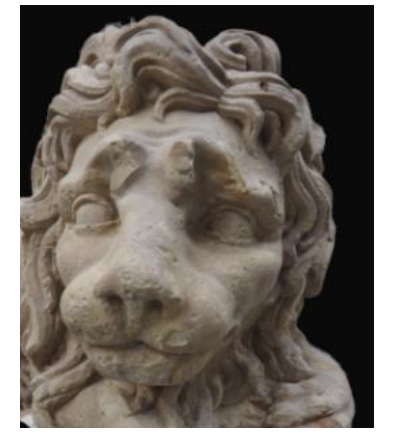

Figure 2. The focused area before filling (Photograph by Sunita Saha)

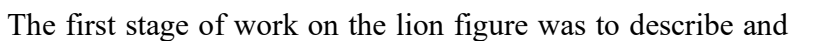

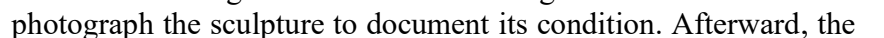
'Lying Lion' was dismantled from its base and transported to the

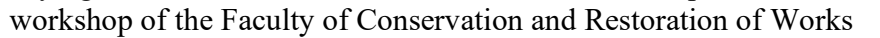

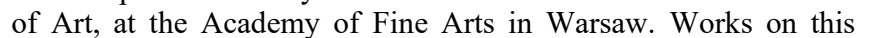

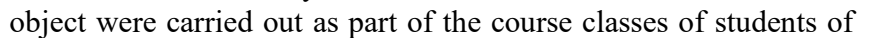
पा

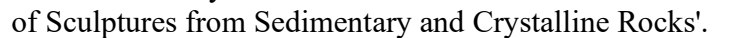

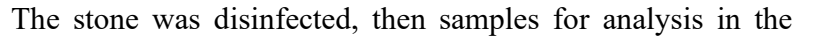

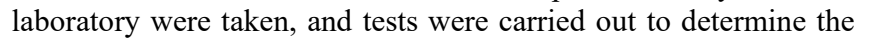

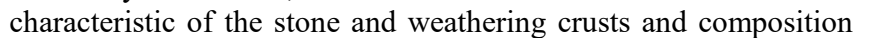

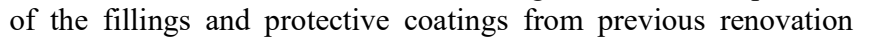
ए

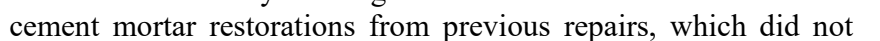

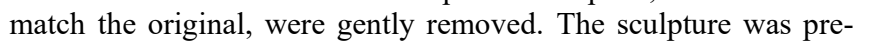
प

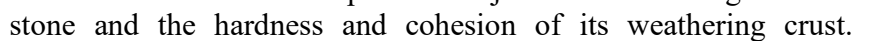

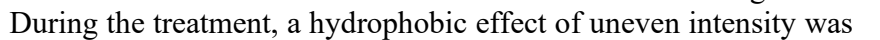

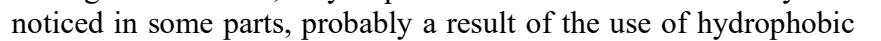

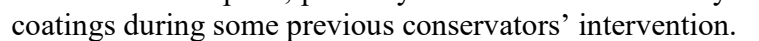

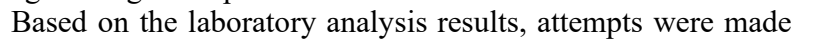

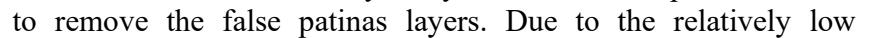

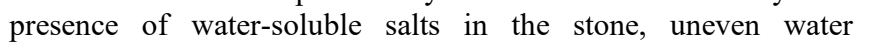

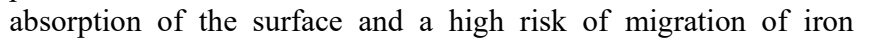

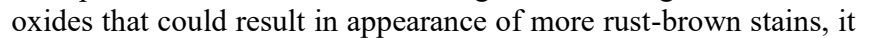

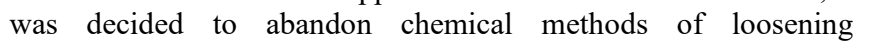

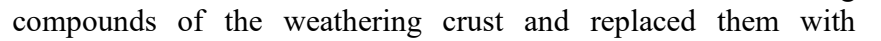

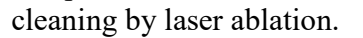

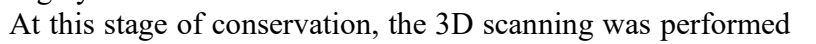

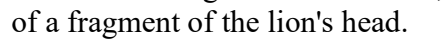

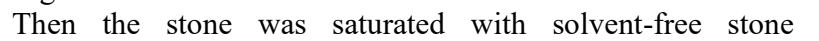

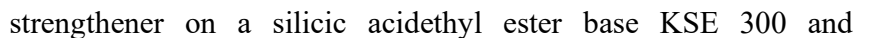

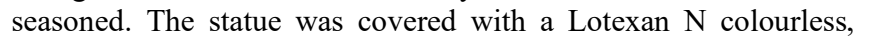

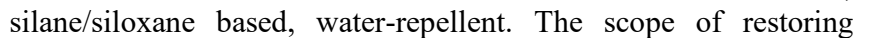

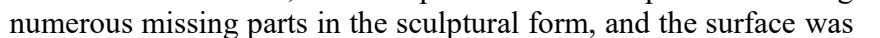

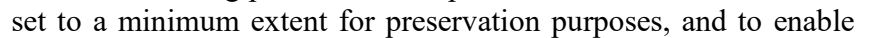

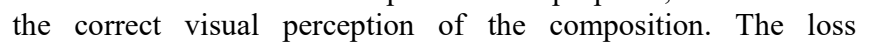

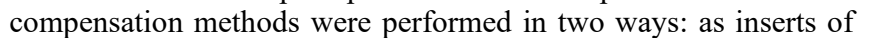

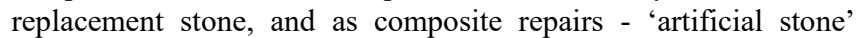

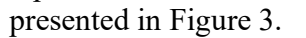

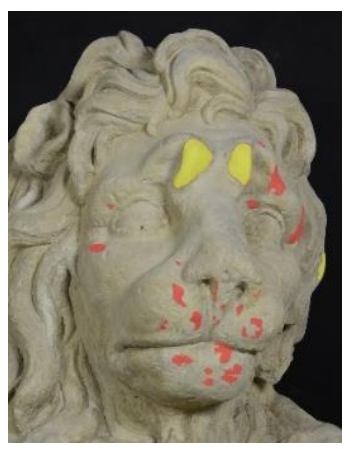

Figure 3: The loss compensation methods: Yellow: insertion of replacement stone, Red: composite repairs (Photograph by Anna Duda-Maczuga)

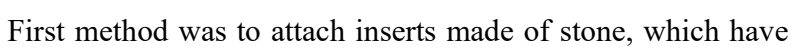

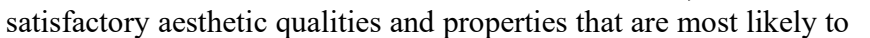

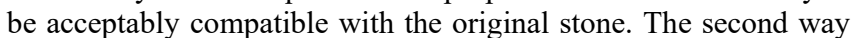

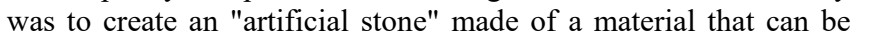

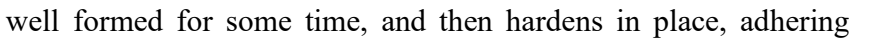

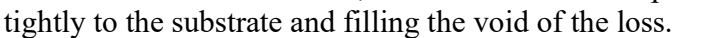

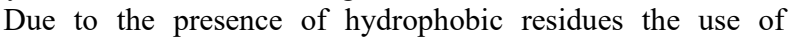

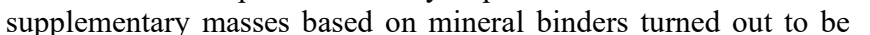

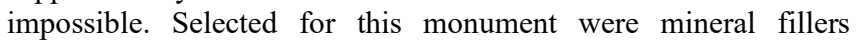

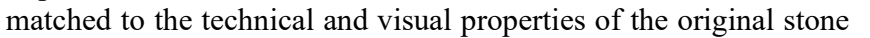

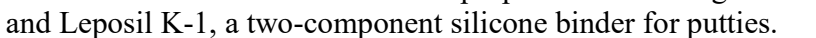

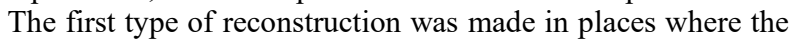
ए

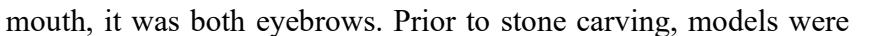

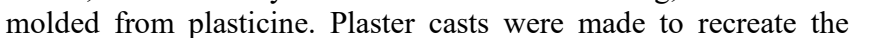

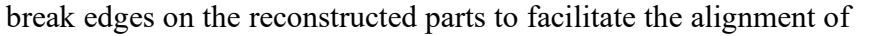

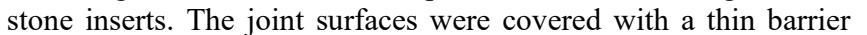

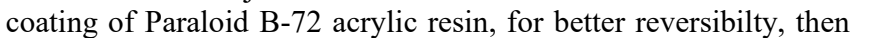

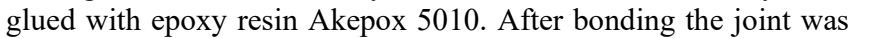

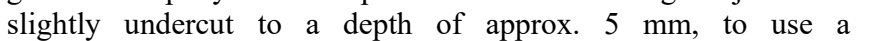

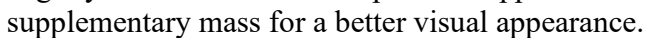

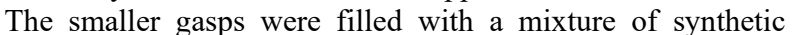

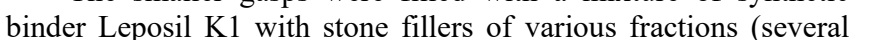

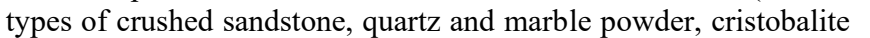
एणाणापणाण

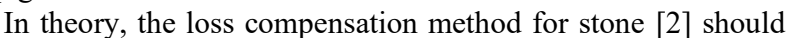

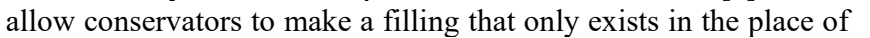

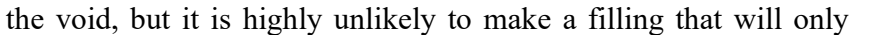

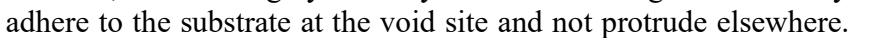

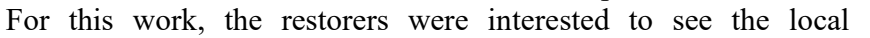

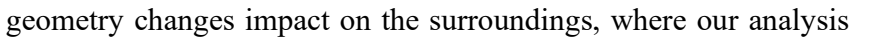

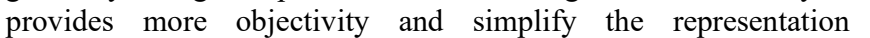

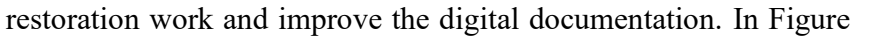

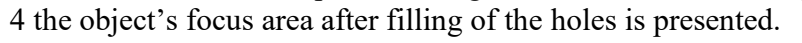

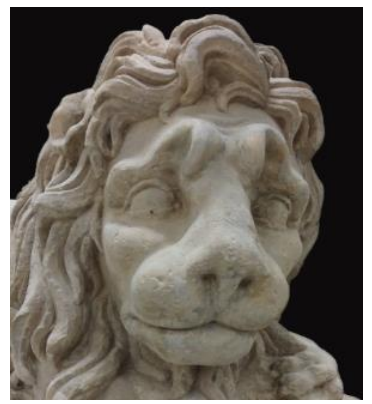

Figure 4. The focused area after filling (Photograph by Anna DudaMaczuga) 


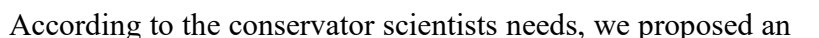

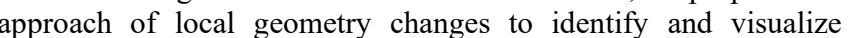

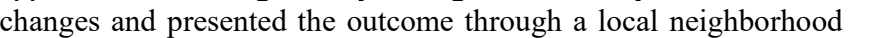

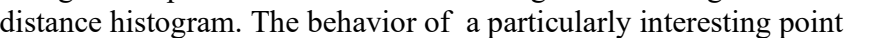

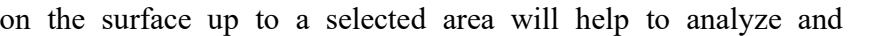

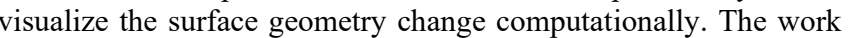

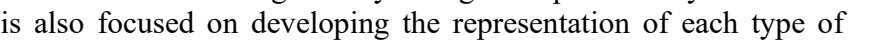

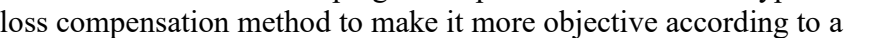

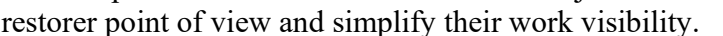

\section{State of the Art}

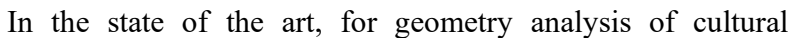

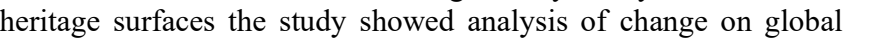

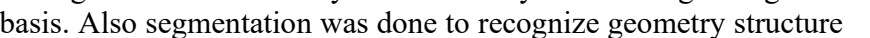

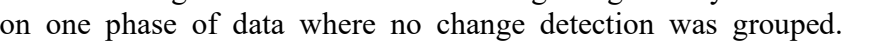

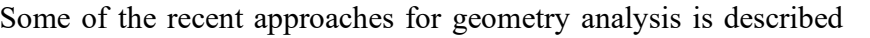

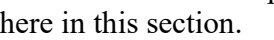

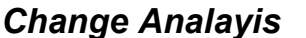

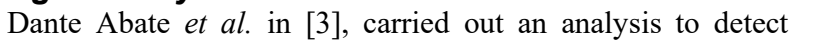

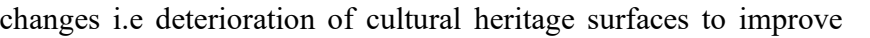

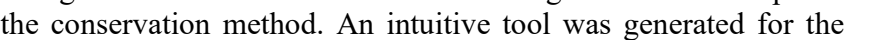

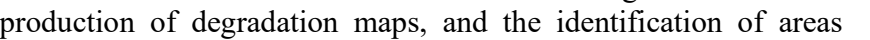

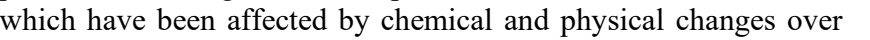

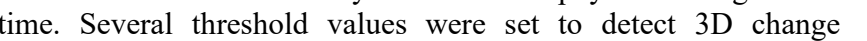

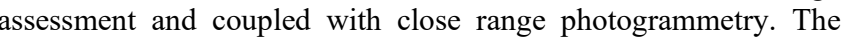

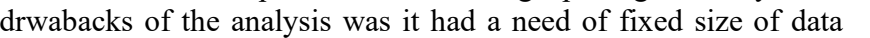

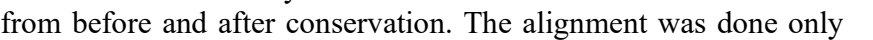

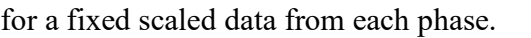

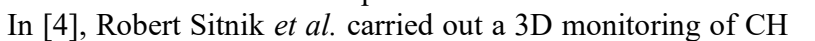

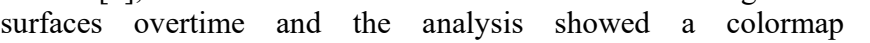

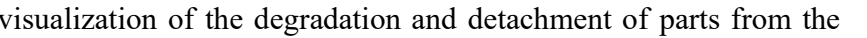

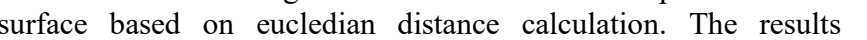

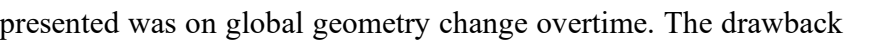

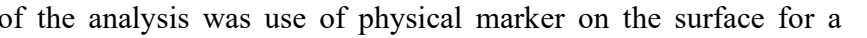

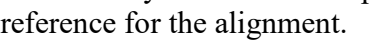

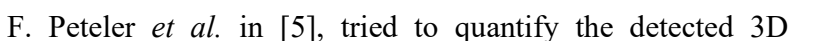

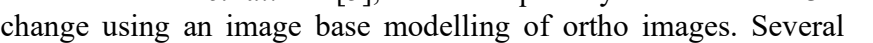

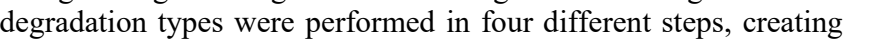

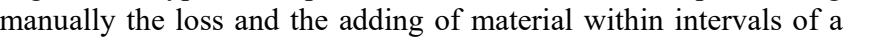

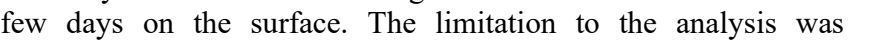

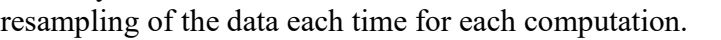

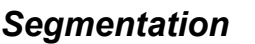

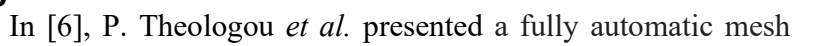

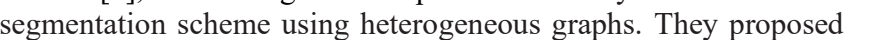

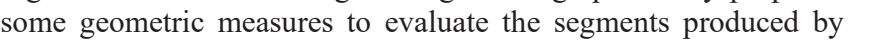

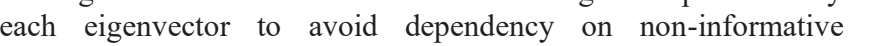

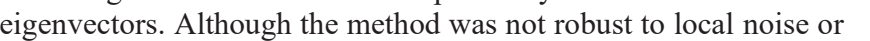

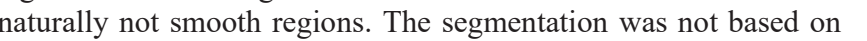

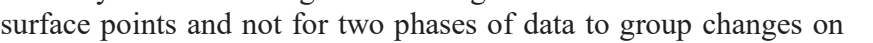
पाणापाण

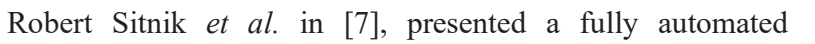

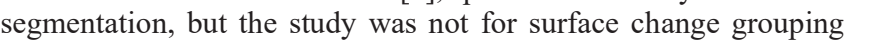
ए

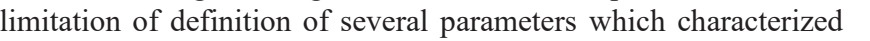
ए ए।

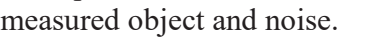

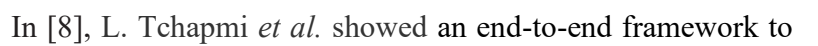

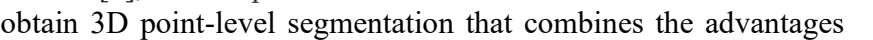

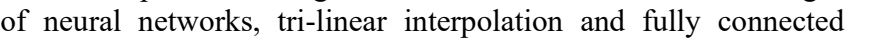

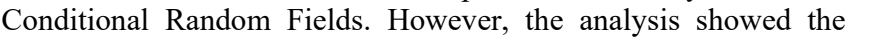

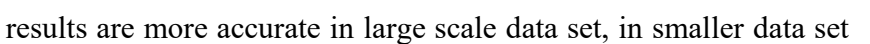

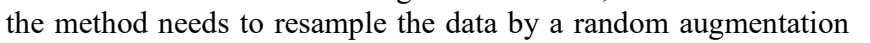

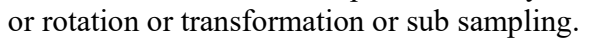

\section{Methodology}

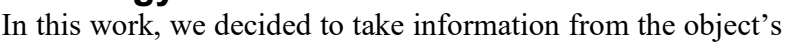

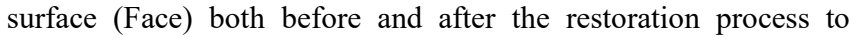

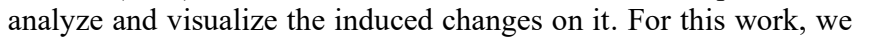

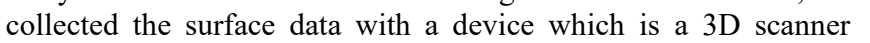

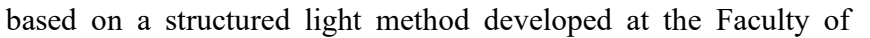

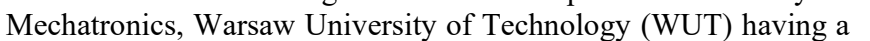

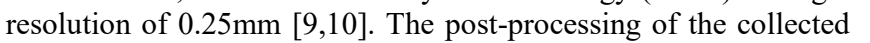

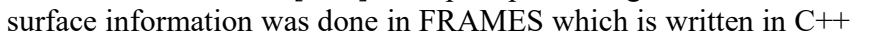

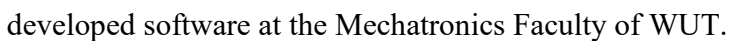

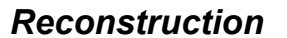

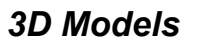

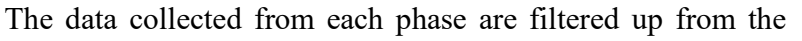

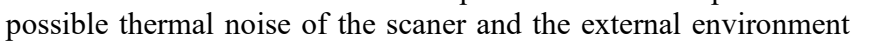

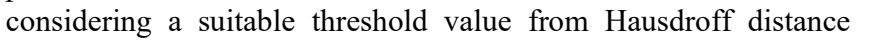

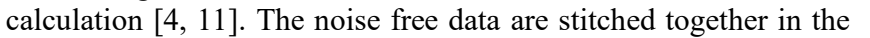

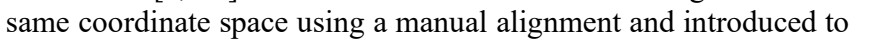

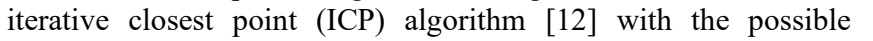

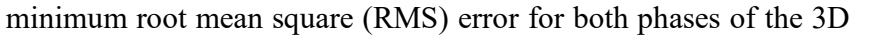
$\square \square \square\|ा\|$
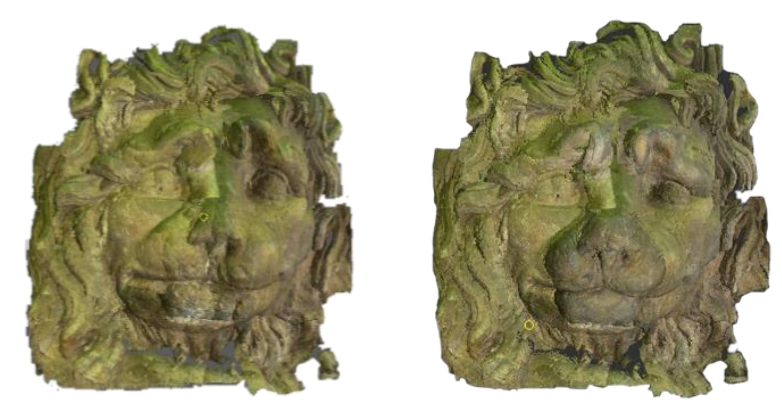

$\square \mathbb{1} \square \quad \square \quad \square \quad \square \quad \square \quad \square \quad \square[\square$

Figure 5: The 3D models (Face) obtained from before(a) and after(b) the restoration process $\square$

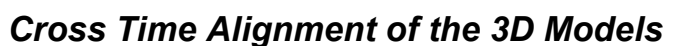

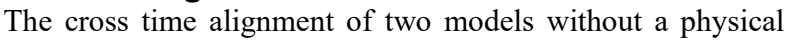

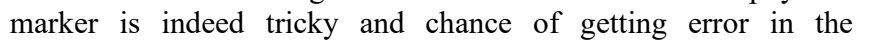

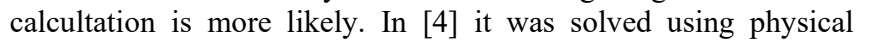

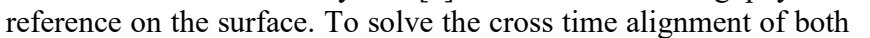
ए

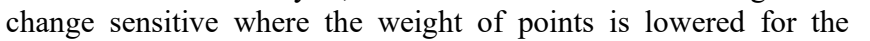

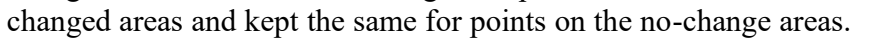

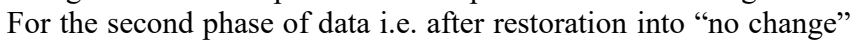

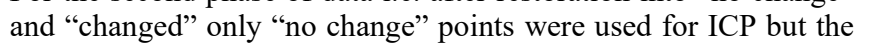

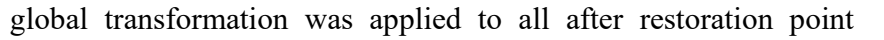
एणाएण

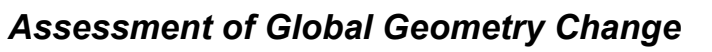

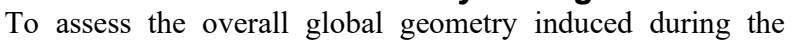

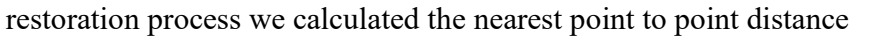

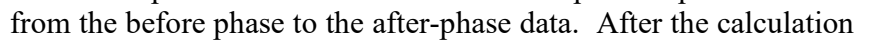

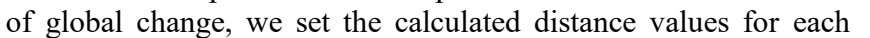

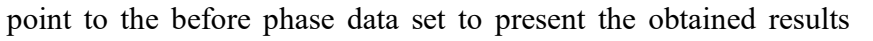

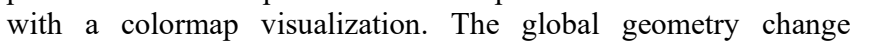
calculation was not enough to present the restorer's work $\square \square \square \square \square \square$ 


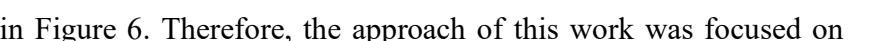

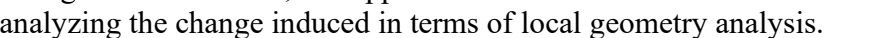

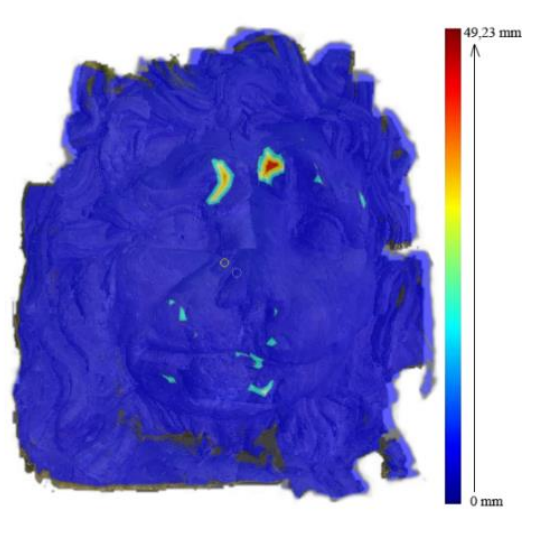

Figure 6: Results obtained from global geometry change analysis

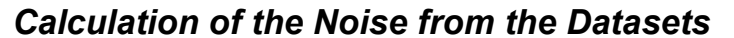

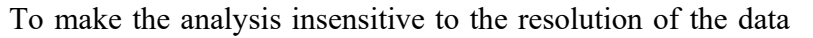

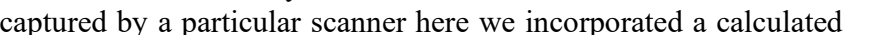

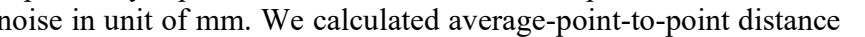

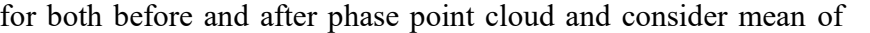

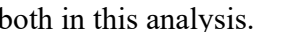

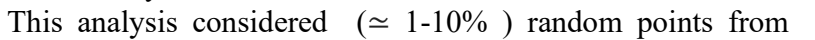

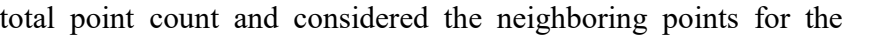

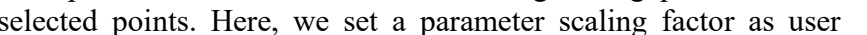

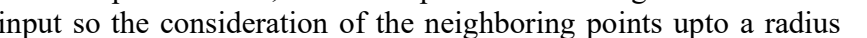

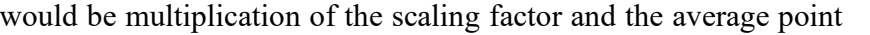

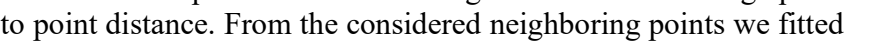

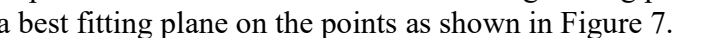

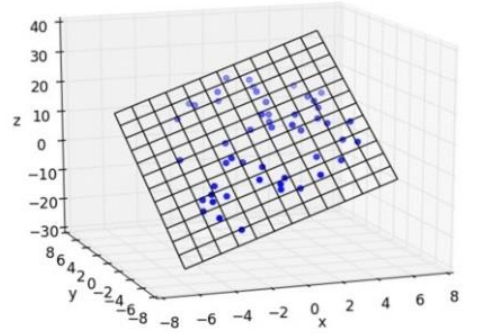

Figure 7: The noise calculation representation $\square$

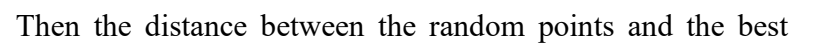

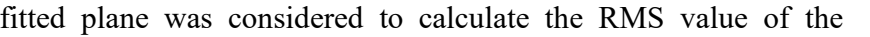

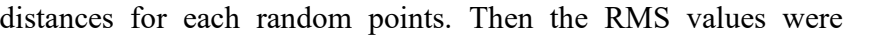

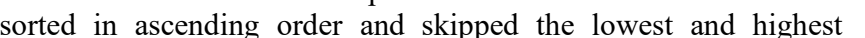

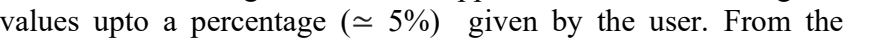

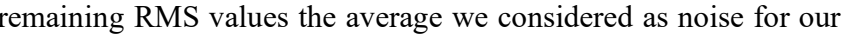

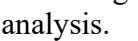

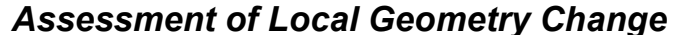

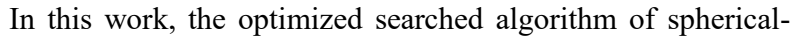

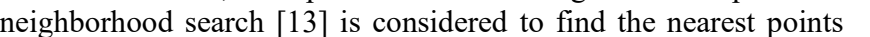

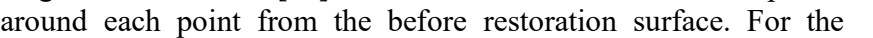

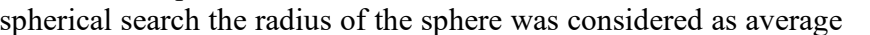

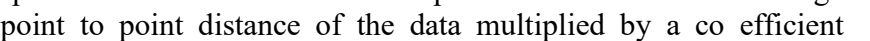

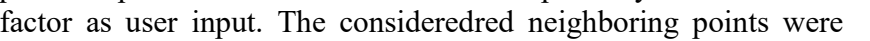

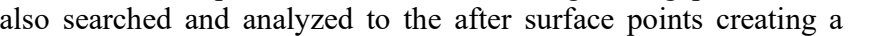

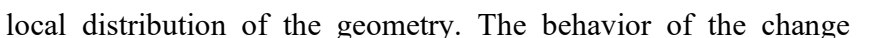

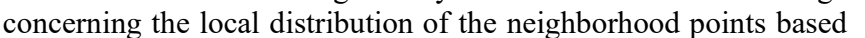

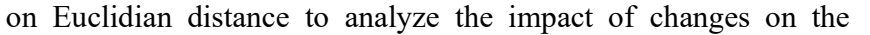

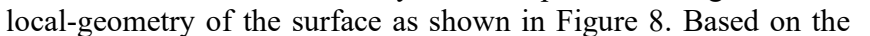

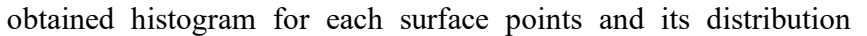

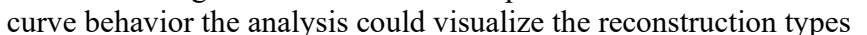

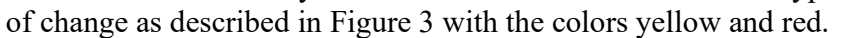

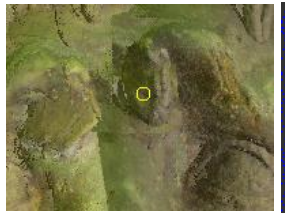

$\square$

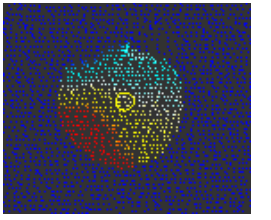

$\square \quad \square \quad \square$
Figure 8: The focus point (a) yellow and obtained spherical neighborhood points and its local geometry behavior (b) $\square$

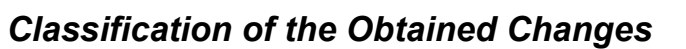

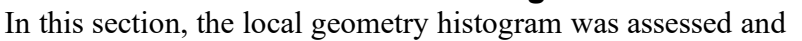

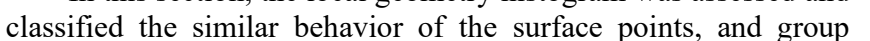

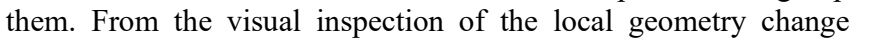

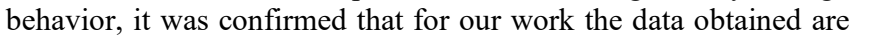

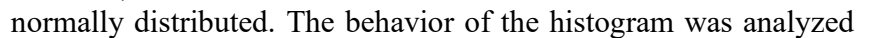

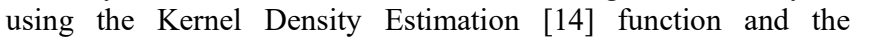

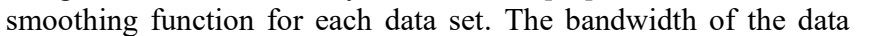

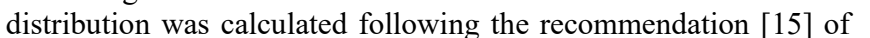

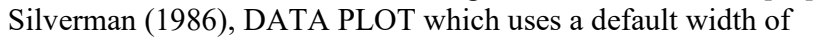

$$
0.9 * \min (\mathrm{s}, \mathrm{IQR} / 1.34) * \mathrm{n}-1 / 5 \square \quad \square \quad \square \quad \square \quad \square \quad \square \mathrm{mm} \square
$$

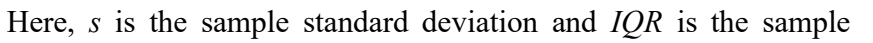

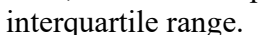

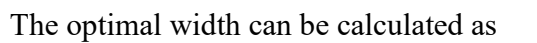

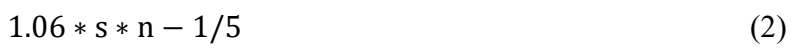

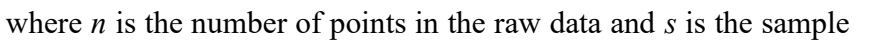

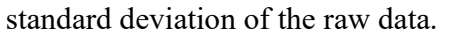

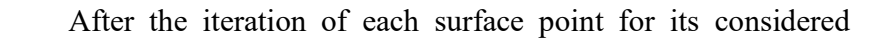

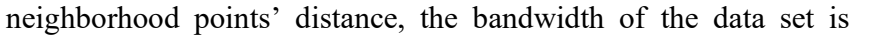

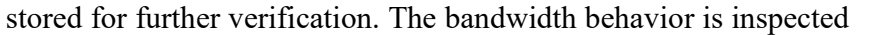

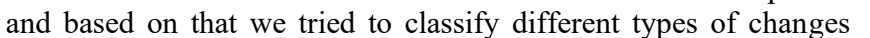

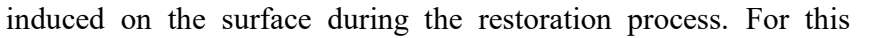

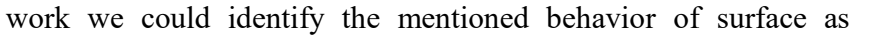

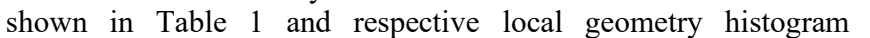

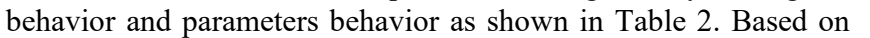

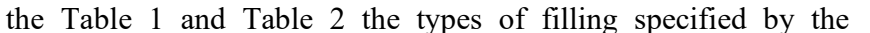

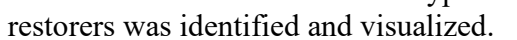

Table 1: Identified surface behavior and local name for the change

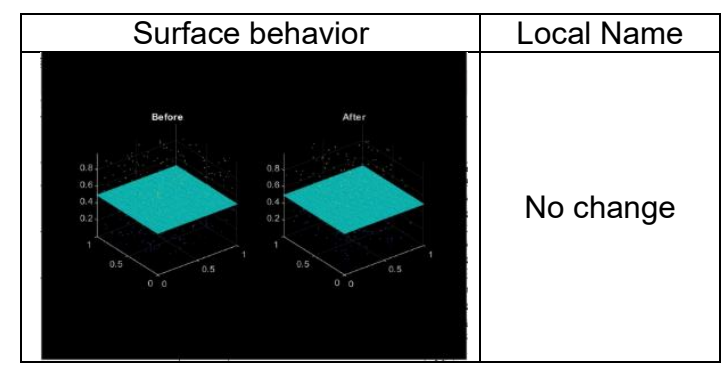

IS\&T International Symposium on Electronic Imaging 2021 3 D Imaging and Applications 2021 


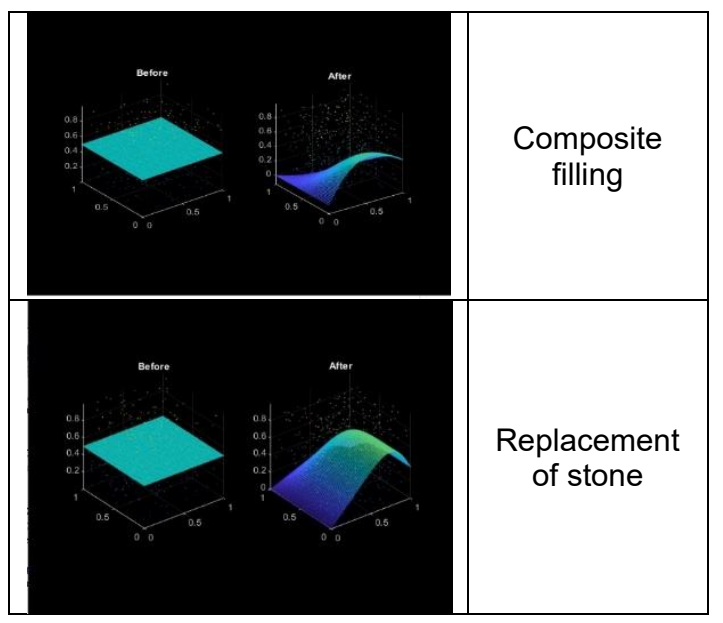

Table 2: Histogram behavior and parameters behavior for the identified changes

\begin{tabular}{|c|c|}
\hline Histogram Behavior & Parameters Behavior \\
\hline 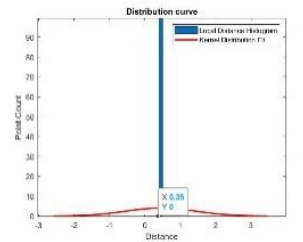 & $\begin{array}{c}\text { bandwidth }=0 \\
\& \& \\
\text { mean }<\text { noise }\end{array}$ \\
\hline 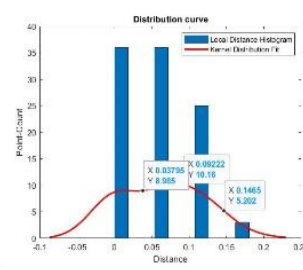 & $\begin{array}{c}\text { bandwidth }<\text { noise } \\
\& \& \\
\text { min(neighbor distance) } \\
<= \\
\text { threshold }\end{array}$ \\
\hline 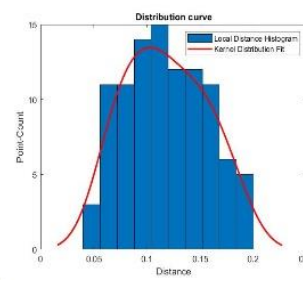 & $\begin{array}{c}\text { bandwidth }<\text { noise } \\
\qquad \& \& \\
\max (\text { neighbor distance) } \\
\sim \\
\text { min(neighbor distance) }\end{array}$ \\
\hline
\end{tabular}

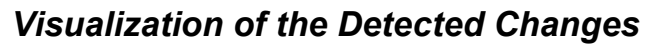

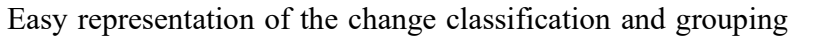

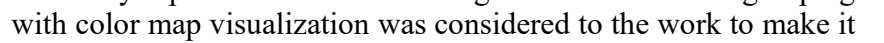

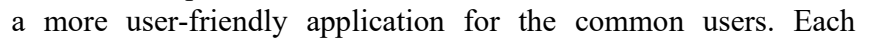
detected type of change was shown on the object's surface with

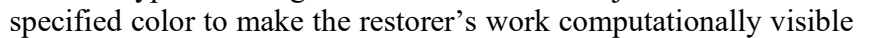

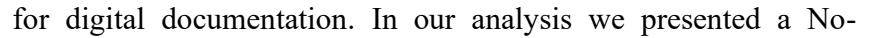

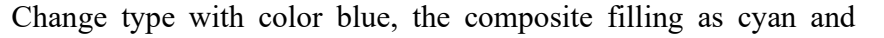

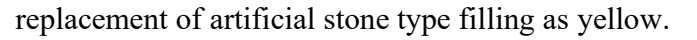

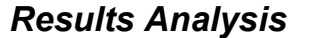

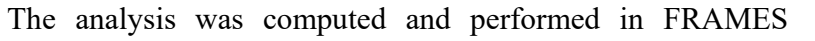

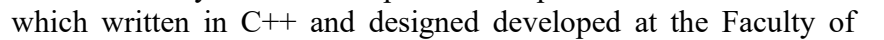

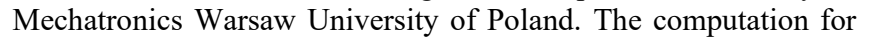

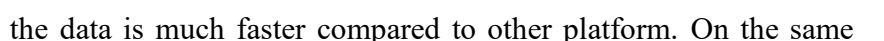

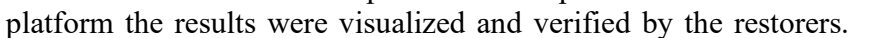

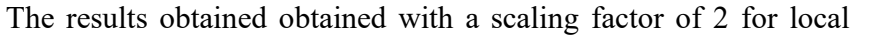

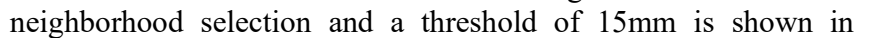

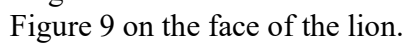

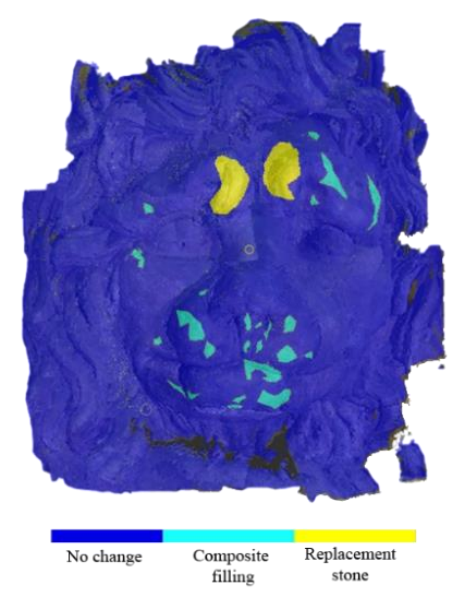

Figure 9: Results obtained from local geometry change analysis shown on the lion face

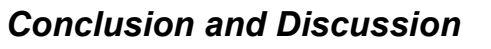

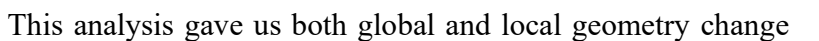

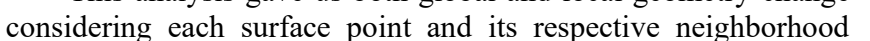

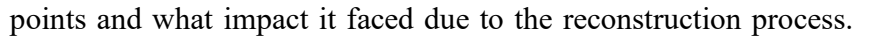

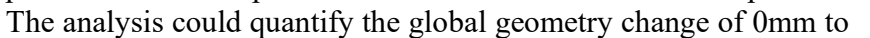

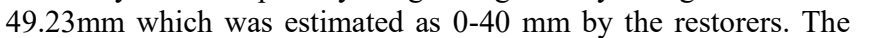

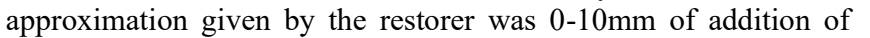

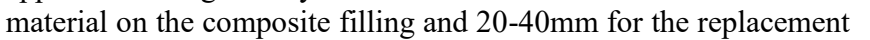

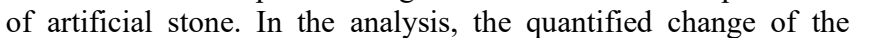

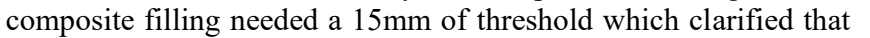
ए।

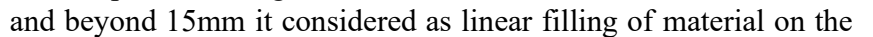

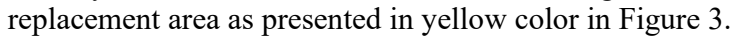

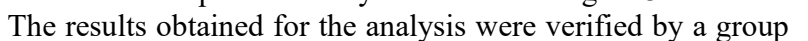

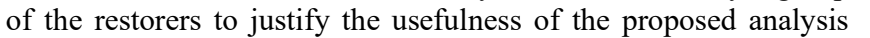

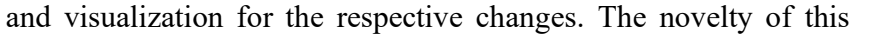

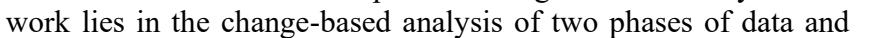

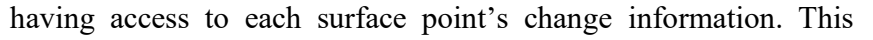

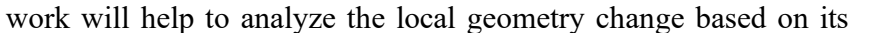

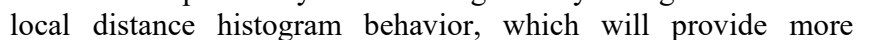

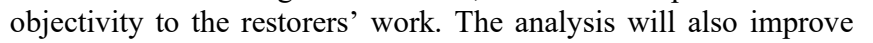

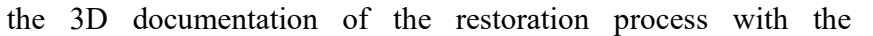

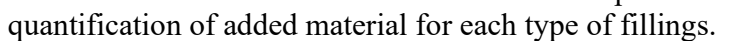

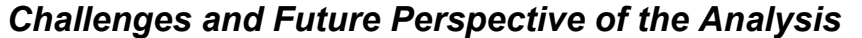 \\ पा

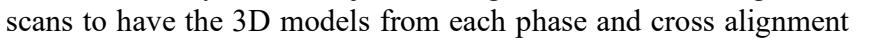

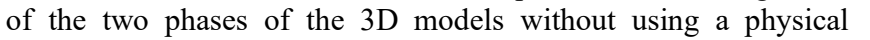

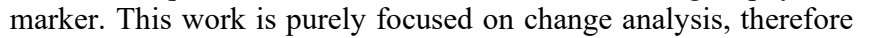

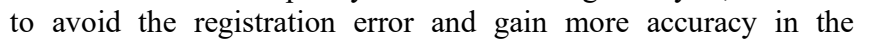

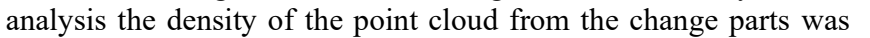

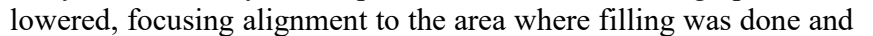

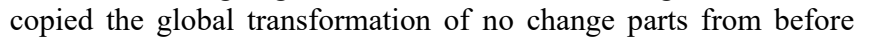

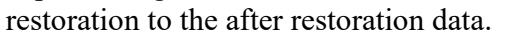

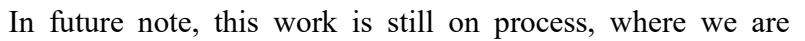

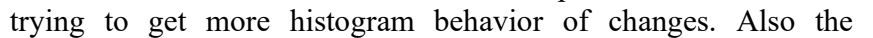

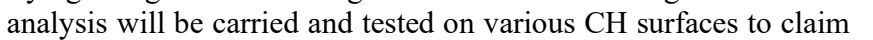




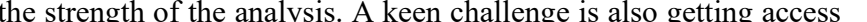

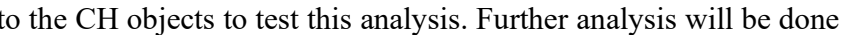

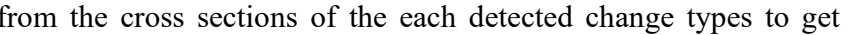

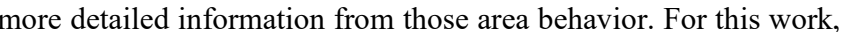

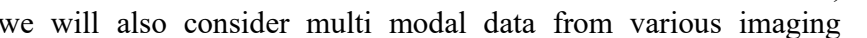

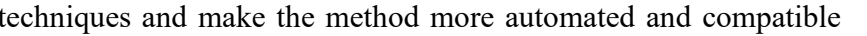

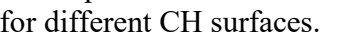

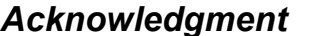

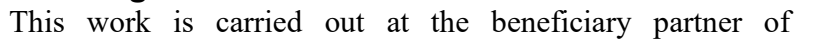 पि口ण Heritage Analysis for New}

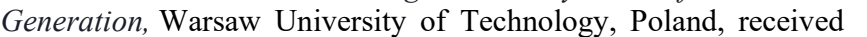
funding from the European Union's Horizon 2020 research and

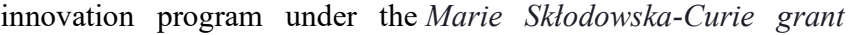

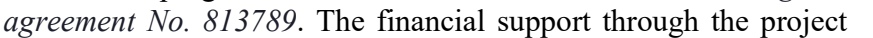

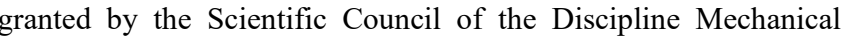

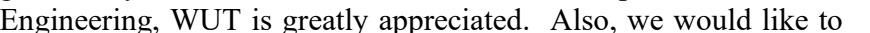
acknowledge Radziwiłł Palace in Nieborów and Romantic Park in

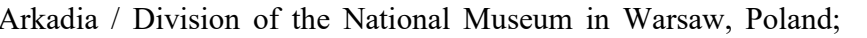

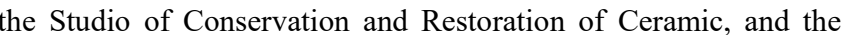

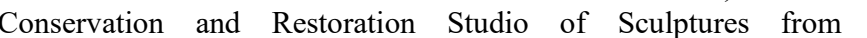

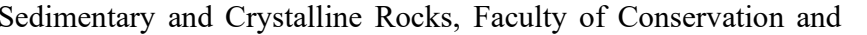

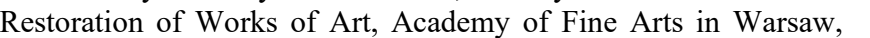

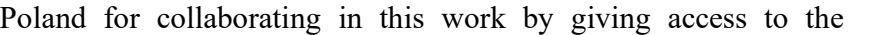

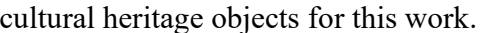

\section{References}

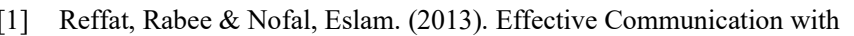

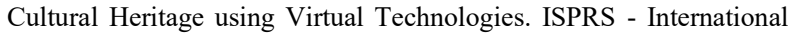

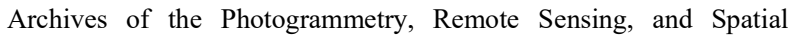

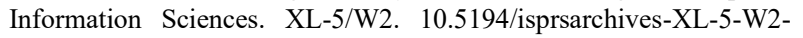

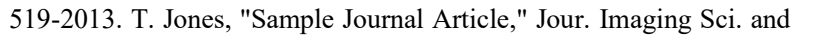

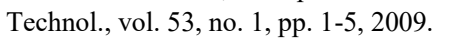

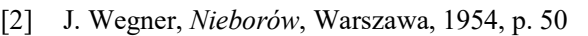

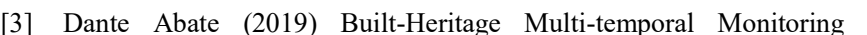

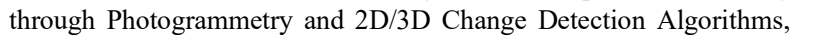

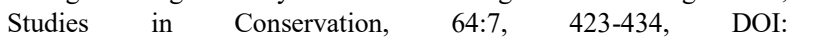

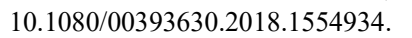

$\square$ R. Sitnik, K. Lech, E. Bunsch, and J. Michoński "Monitoring surface

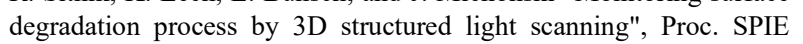

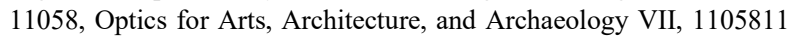

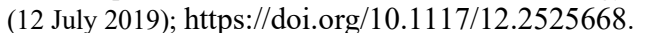

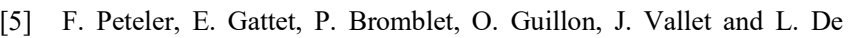

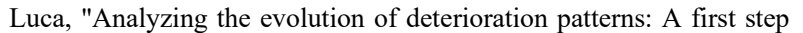

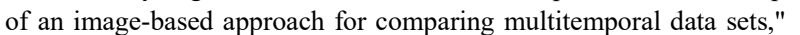

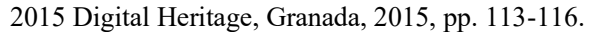

(I)

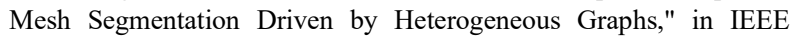

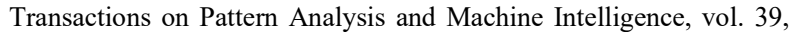

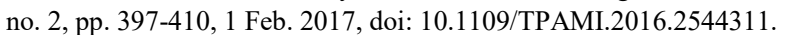

पा

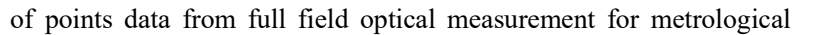

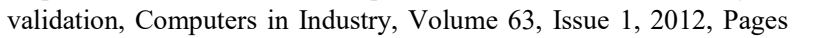

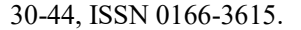

ए

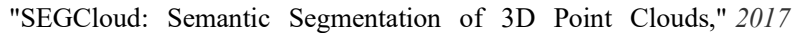

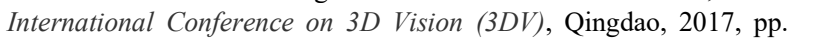
पाIणाणा $\square \square$ J. Geng, "Structured light 3D surface imaging: a tutorial", Advances

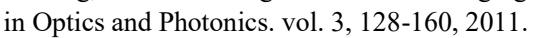

$\square \square$ M. Adamczyk, M. Kamiński, R. Sitnik, A. Bogdan, M. Karaszewski,

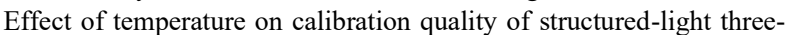

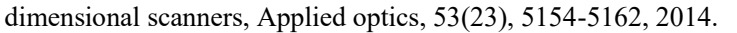

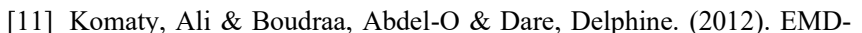

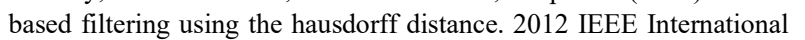

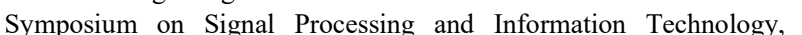

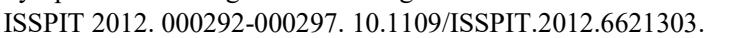

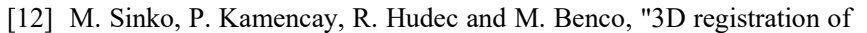

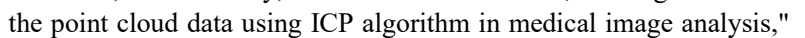

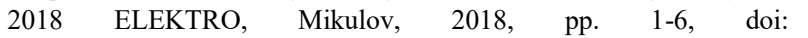

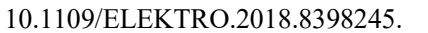

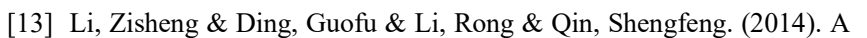

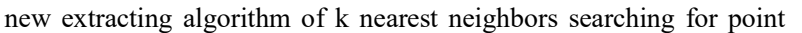

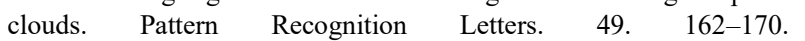

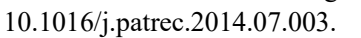

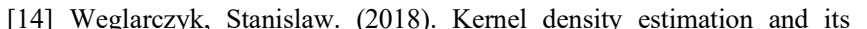

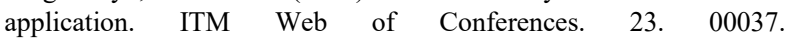

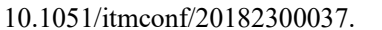

$\square \square$ B.W. Silverman, "DENSITY ESTIMATION FOR STATISTICS AND DATA ANALYSIS", Published in Monographs on Statistics

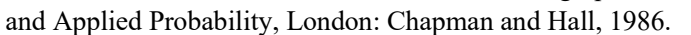

\section{Author Biography}

Sunita Saha received her master's degree in computer science and engineering from NERIST (2018). She is currently making her doctoral studies at the Faculty of Mechatronics Warsaw University of Technology. Also, a Marie Curie ITN fellow (ESR4) at the EU project CHANGE. Her works focus on the Analysis and visualization of multi-modal image data in $\mathrm{CH}$ surface monitoring over time or before and after the restoration process.

Anna Duda-Maczuga received her master's degree in conservation and restoration of stone sculpture and carved architectural in Academy of Fine Arts in Warsaw, where she is currently an assistant at the Conservation and Restoration Studio of Sculptures from Sedimentary and Crystalline Rocks. The area of her interest is the use of modern research methods in heritage protection.

Athanasia Papanikolaou received her master's in Photonics and Nanoelectronics from the University of Crete. Currently she is a PhD Candidate at Warsaw University of Technology and a Marie Curie ITN fellow at the EU project CHANGE. Her work is focused on the development of a portable multimodal device for surface analysis and monitoring of cultural heritage objects.

Robert Sitnik (Member of OSA and SPIE) received his MSc Eng (1999), PhD (2002) in applied optics from the Warsaw University of Technology. He has authored and co-authored more than hundred scientific papers. His interests are structured light shape measurement $(3 D / 4 D)$, triangulation methods, digital image processing, computer graphics, animation software development and virtual reality techniques. He has been a leader of projects from various fields like $3 D$ optical metrology, virtual and augmented reality and supporting cultural heritage by opto-numerical solutions. He is head of Virtual Reality Techniques Division at WUT. 


\section{JOIN US AT THE NEXT EI!}

IS\&T International Symposium on

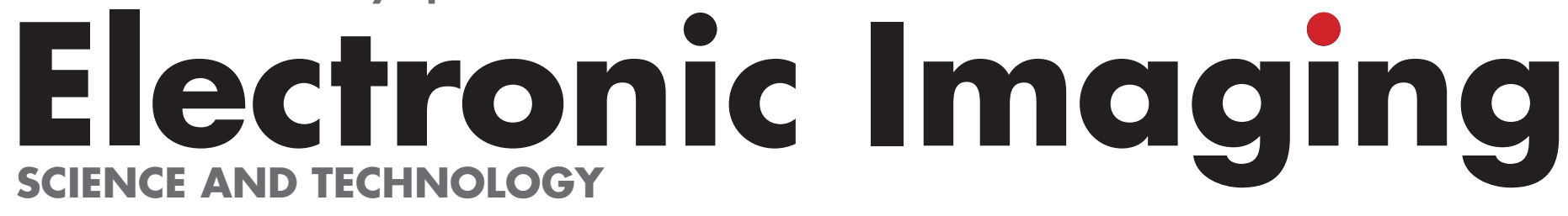

Imaging across applications ... Where industry and academia meet!
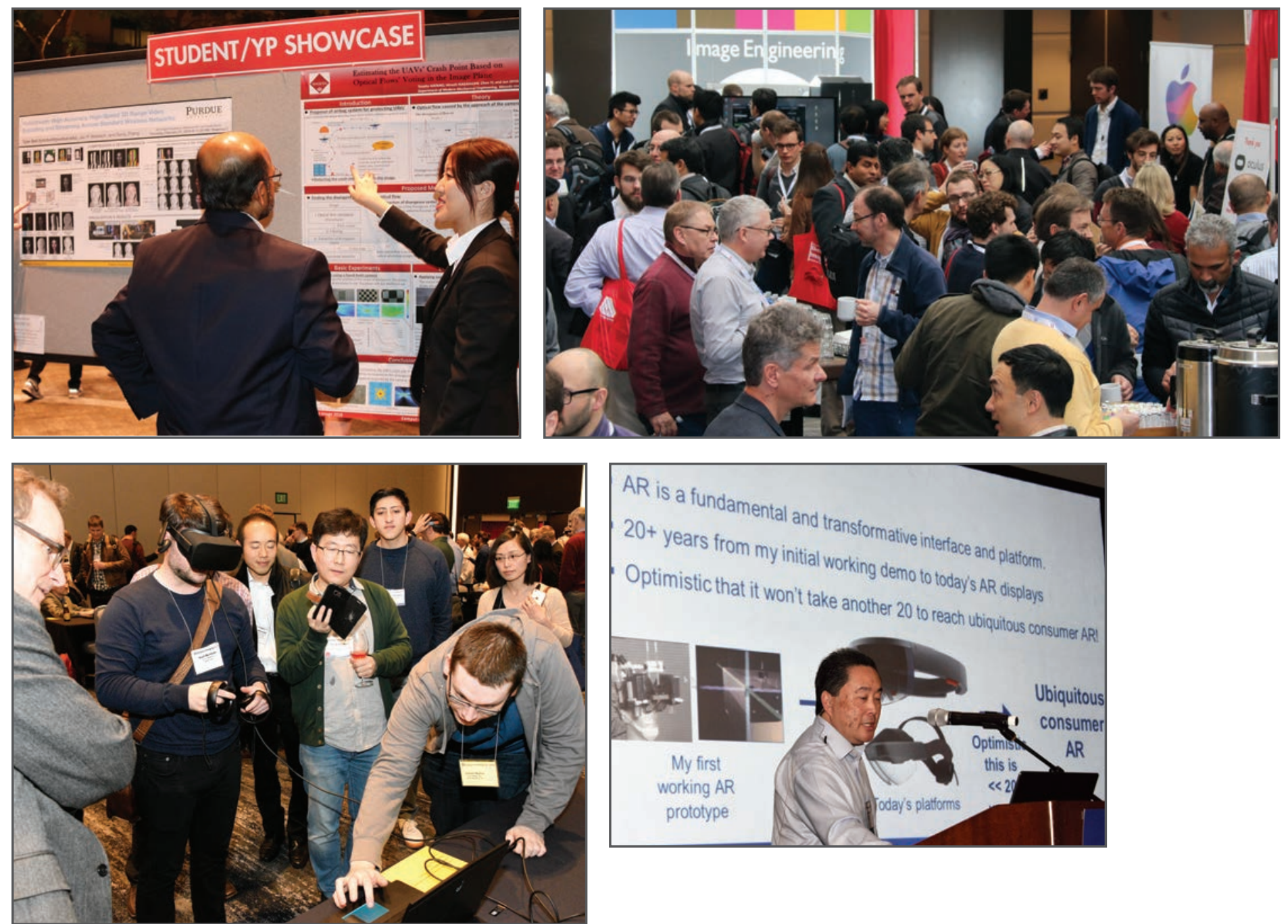

- SHORT COURSES • EXHIBITS • DEMONSTRATION SESSION • PLENARY TALKS •

- INTERACTIVE PAPER SESSION • SPECIAL EVENTS • TECHNICAL SESSIONS •

www.electronicimaging.org

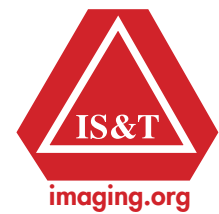

